\title{
A selenocysteine tRNA and SECIS element in Plasmodium falciparum
}

\author{
TOBIAS MOURIER, ARNAB PAIN, BART BARRELL, and SAM GRIFFITHS-JONES
}

The Wellcome Trust Sanger Institute, Wellcome Trust Genome Campus, Hinxton, Cambridge CB10 1SA, United Kingdom

\begin{abstract}
The molecular machinery for incorporating selenocysteine into proteins is present in both prokaryotes and eukaryotes. Although selenocysteine insertion has been reported in animals, plants, and protozoans, known eukaryotic selenocysteine tRNA sequences and selenocysteine insertion sequences are limited to animals and plants. Here we present clear indications of the presence of selenocysteine-tRNA and a selenocysteine insertion sequence in Plasmodium falciparum. To our knowledge, this is the first report of an identification of protozoan selenocysteine insertion machinery at the sequence level.
\end{abstract}

Keywords: Plasmodium; malaria; apicomplexa; selenocysteine; SECIS element

Systematic incorporation of selenium into protein is facilitated by a tRNA on which the initial serine is converted to selenocysteine (hence designated $\mathrm{tRNA}^{[\mathrm{Ser}] \mathrm{Sec}}$ ). In the presence of a selenocysteine insertion sequence (SECIS) in the mRNA, the tRNA ${ }^{[\mathrm{Ser}] \mathrm{Sec}}$ recognizes UGA that otherwise acts as a stop codon. Selenoproteins are most often involved in redox regulation of metabolic processes; disruption of eukaryotic selenocysteine insertion is in several cases reported to be lethal to the organism (Bosl et al. 1997; Kohrle et al. 2000). Eukaryotic proteins with incorporated selenocysteines have been found in animals and in a single representative from plants (Fu et al. 2002; Novoselov et al. 2002) and protozoans (Osaka et al. 2003), respectively.

From the genome of the apicomplexan malaria parasite Plasmodium falciparum (Gardner et al. 2002) we have identified a putative $\mathrm{tRNA}^{[\mathrm{Ser}] \mathrm{Sec}}$. This prompted a search for other indications of the presence of selenocysteine insertion machinery, which revealed a SECIS-like sequence, conserved across Plasmodium phylogeny, and with covariation indicative of a conserved secondary structure. The $P$. falciparum SECIS element is situated downstream of MAL8P1.86, a protein of unknown function, in which the presence of a putative TGA selenocysteine codon has caused gene prediction algorithms to incorrectly identify a 60-bp intron covering the TGA codon.

Reprint requests to: Tobias Mourier, The Wellcome Trust Sanger Institute, Wellcome Trust Genome Campus, Hinxton, Cambridge CB10 1SA, United Kingdom; e-mail: tmourier@bi.ku.dk; fax: 44-1223-494919.

Article and publication are at http://www.rnajournal.org/cgi/doi/ 10.1261/rna.7185605.
A possible selenocysteine tRNA was identified by a whole genome analysis of $(\mathrm{G}+\mathrm{C})$-rich regions in $P$. falciparum. This was supported by tRNAscan-SE prediction (Lowe and Eddy 1997) using the covariance model analysis only for maximum sensitivity. Running tRNAscan-SE at the default setting did not result in any tRNA predictions, indicating a divergent primary structure. Homologs of the putative tRNA $^{[\text {Ser]Sec }}$ were detectable in a range of other Plasmodium genomes (Figs. 1, 2). We also detected Sec-tRNAs in Toxoplasma gondii and Eimeria tenella (Figs. 1, 2), but not in Theileria annulata or Cryptosporidium parvum. The putative apicomplexan Sec-tRNAs display the atypically long vari-

\begin{tabular}{|c|c|c|c|}
\hline & tRNA $A^{|s e r| k c e}$ & SECIS element & $\begin{array}{l}\text { MAL8P1.86 } \\
\text { homologue }\end{array}$ \\
\hline Plasmodium falciparum & + & + & + \\
\hline -Plasmodium reichenewi & + & nd & nd \\
\hline LPlasmodiun gallinaceum & + & + & + \\
\hline -Plasmodiun knowlesi & + & + & + \\
\hline Plasmodium berghei & + & + & nd \\
\hline LPlasmodium yoelii & + & + & + \\
\hline LPlasmodiun chabaudi & + & + & + \\
\hline$\Gamma^{\text {Toxoplasma gondii }}$ & + & nd & nd \\
\hline Limeria tenelia & + & nd & nd \\
\hline L-Theileria annulata & nd & nd & nd \\
\hline Cryptosporidium parvum & nd & nd & nd \\
\hline
\end{tabular}

FIGURE 1. Presence of detectable components in apicomplexan genomes. Evolutionary relationship is indicated on the left (Escalante and Ayala 1994; Barta et al. 2001). (nd) Not detectable. Sequence sources: Published genomes of P. falciparum, P. yoelii, and C. parvum appear in Gardner et al. (2002), Carlton et al. (2002), and Abrahamsen et al. (2004), respectively; unfinished genomes of $P$. berghei, $P$. chabaudi, $P$. gallinaceum, P. knowlesi, $P$. reichenowi, E. tenella, and T. annulata are provided by The Pathogen Sequencing Unit, The Wellcome Trust Sanger Institute (http://www.sanger.ac.uk/) and GeneDB (http://www.genedb.org/); draft genome sequence of T. gondii was searched at ToxoDB (http://toxodb.org/). 

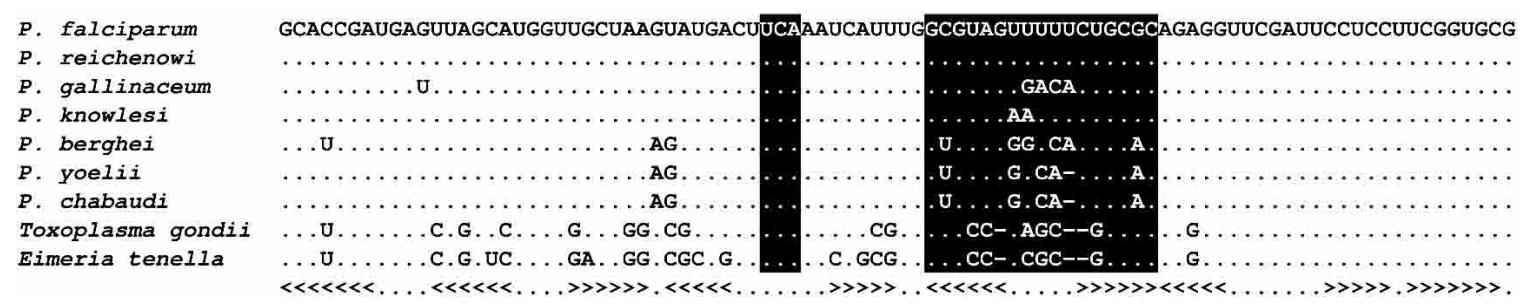

FIGURE 2. Alignment of putative Sec-tRNAs. The predicted secondary structure consensus is shown below as nested parentheses. Only residues differing from the $P$. falciparum sequence are shown. Identical residues indicated as dots, gaps as dashes. The UCA anticodon and the long variable arm are highlighted.

able arm and a resulting length of around 90 nt previously seen in other eukaryotic tRNA ${ }^{[\mathrm{Ser}] \mathrm{Sec}}$ (Hatfield and Gladyshev 2002). The $P$. falciparum $\mathrm{tRNA}^{[\mathrm{Ser}] \mathrm{Sec}}$ is flanked by a Met-tRNA and the gene encoding PF14_0361 on chromosome 14. Although data are limited by the unfinished nature of the genomes, this region appears to have conserved synteny in Plasmodium species (data not shown).

In a previous study, Lambert and coworkers published a list of confirmed and putative SECIS elements identified using the ERPIN program (Gautheret and Lambert 2001; Lambert et al. 2002). This computational prediction included three low-scoring SECIS elements from $P$. yoelii and P. vivax. WU-BLAST (http://blast.wustl.edu/) searches with the $P$. yoelii sequence returned significant matches from a range of other Plasmodium genomes (Fig. 1). An alignment of the putative SECIS elements is shown in Figure 3. The elements display three helices, hence conforming to the Form 2 SECIS element structure (for review, see Krol 2002). A conventional eukaryotic non-Watson-Crick quartet (UGAN-NGAN, highlighted in Fig. 3) preceded by adenosine is conserved in all candidate sequences. Also, the invariant AA pair in the internal loop 2 is conserved (Korotkov et al. 2002).

The putative $P$. falciparum SECIS element is situated 134 bp downstream of the MAL8P1.86 gene. The translated product of this gene has no annotated function and does not contain any known protein domains (Pfam15: Bateman et al. 2004). Microarray analysis has shown up-regulation of MAL8P1.86 expression during the $P$. falciparum trophozoite stage in human red blood cells (Bozdech et al. 2003). The MAL8P1.86 gene is annotated as having a 60-bp intron in which the TGA codon resides (GeneDB, http://www. genedb.org/). The TGA codon is conserved in Plasmodium genomes as is the coding potential of the entire intron (Fig. 4). The possibility of a highly conserved intron is rendered unlikely by the finding that the splice signals are not detected outside P. falciparum (Fig. 4). Hence, rather than being an intron, this sequence most likely represents the part of the coding sequence containing a selenocysteine codon. The presence of selenocysteine insertion in $P$. falciparum is further supported by the fact that the P. falciparum genome encodes a putative selenocysteine-specific elongation factor and a putative selenophosphate synthetase (PFA0495c and PFI0505, respectively).

The SECIS element from P. berghei resides in the very proximal end of a contig, and the absence of a MAL8P1.86 homolog is most likely due to low genome coverage (Fig. 1). Given the close relationship between $P$. falciparum and $P$. reichenowi, the fact that neither the SECIS element nor any MAL8P1.86 homolog is detectable in the latter (Fig. 1) is most easily explained by low coverage (consistent with the $\sim 1 \times$ coverage of the $P$. reichenowi genome). However, we cannot rule out the possibility that these features have been recently lost (within the last $\sim 10$ million years; Rich and



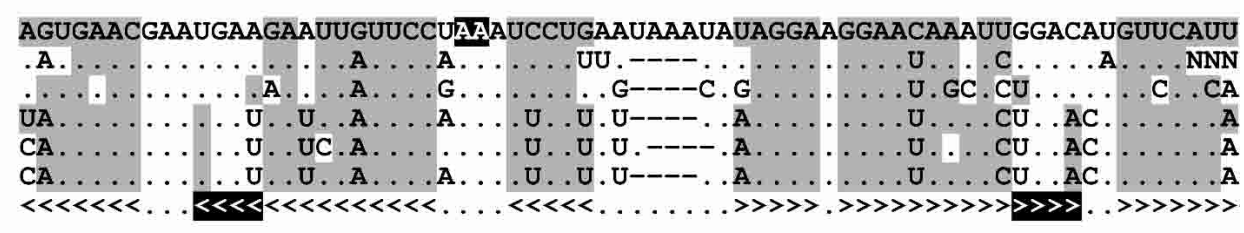

B

FIGURE 3. (A) Alignment of putative SECIS elements. Only residues differing from the $P$. falciparum sequence are shown. Gray-shaded residues are able to base pair in accordance with the predicted consensus secondary structure shown below. $(B)$ Predicted secondary structure for the putative P. falciparum SECIS element. The non-Watson-Crick quartet and the invariant AA pair (Korotkov et al. 2002; Krol 2002) are denoted by black boxes and bold letters in alignment and structure, respectively. 



FIGURE 4. Alignment of genomic regions with a predicted selenocysteine codon. Genomic DNA (top) from five Plasmodium species. Only residues differing from the $P$. falciparum sequence are shown. Annotated intron in $P$. falciparum is in lower case. Note that the suggested donor and acceptor sites are not conserved. Translated protein sequences are shown below. The TGA codon (highlighted) is translated as selenocysteine $(\mathrm{U})$.

Ayala 2000) and that the P. reichenowi Sec-tRNA is conserved for the processing of another selenoprotein (see below).

Searching the complete $P$. falciparum genome with covariance models built from alignments of both animal SECIS elements and the Plasmodium sequences identified here did not suggest further convincing SECIS candidates (Infernal: http://www.genetics.wustl.edu/eddy/infernal/, Eddy 2002; Griffiths-Jones et al. 2003). Hence, if P. falciparum hosts further SECIS elements, these will be divergent in sequence and secondary structure. The human genome is reported to contain 25 selenoproteins (Kryukov et al. 2003), but as Caenorhabditis elegans appears to only encode a single selenoprotein (Gladyshev et al. 1999), this would not be unprecedented in eukaryotic genomes.

Given the involvement of selenoproteins in redox regulation and the up-regulation of MAL8P1.86 during a Plasmodium stage in human blood cells, it is tempting to speculate that the Plasmodium selenoprotein may have a role in metabolic processes related to redox regulation within infected red blood cells. It may not be coincidental that MAL8P1.86 is located next to a superoxide dismutase (SOD) gene. The Plasmodium selenoprotein —which unlike the majority of known eukaryotic selenoproteins is not widely conserved-may play a part in a novel and vital SOD-related pathway. Identification of this malarial selenoprotein may lead to new areas of malaria biology yet to be explored. However, until more is known about the exact function of MAL8P1.86 this remains purely conjectural.

As ab initio gene prediction algorithms will interpret any TGA selenocysteine codon as a stop codon, selenoproteins are likely to be missed in genome annotations. Therefore, selenoprotein detection requires more detailed analysis, including direct experimental detection of incorporated selenium (Gladyshev et al. 1999; Novoselov et al. 2002; Osaka et al. 2003), structural analysis of SECIS structures (Lambert et al. 2002; Kryukov et al. 2003), or-as in the present study-a comparative analysis of related genomes. With the above in mind, it is likely that reports of protozoan selenoproteins will continue to appear, perhaps rendering system- atic selenocysteine insertion more the rule than the exception in the tree of life.

\section{ACKNOWLEDGMENTS}

T.M. was supported by a grant from The Carlsberg Foundation. Work at The Sanger Institute is funded by The Wellcome Trust.

Received September 22, 2004; accepted November 23, 2004.

\section{REFERENCES}

Abrahamsen, M.S., Templeton, T.J., Enomoto, S., Abrahante, J.E., Zhu, G., Lancto, C.A., Deng, M., Liu, C., Widmer, G., Tzipori, S., et al. 2004. Complete genome sequence of the apicomplexan, Cryptosporidium parvum. Science 304: 441-445.

Barta, J.R., Martin, D.S., Carreno, R.A., Siddall, M.E., ProfousJuchelkat, H., Hozza, M., Powles, M.A., and Sundermann, C. 2001. Molecular phylogeny of the other tissue coccidia: Lankesterella and Caryospora. J. Parasitol. 87: 121-127.

Bateman, A., Coin, L., Durbin, R., Finn, R.D., Hollich, V., GriffithsJones, S., Khanna, A., Moxon, S., Sonnhammer, E.L.L., Studholme, D.J., et al. 2004. The Pfam protein families database. Nucleic Acids Res. 32: D138-D141.

Bosl, M.R., Takaku, K., Oshima, M., Nishimura, S., and Taketo, M.M. 1997. Early embryonic lethality caused by targeted disruption of the mouse selenocysteine tRNA gene (Trsp). Proc. Natl. Acad. Sci. 94: 5531-5534.

Bozdech, Z., Llinas, M., Pulliam, B.L., Wong, E.D., Zhu, J., and DeRisi, J.L. 2003. The transcriptome of the intraerythrocytic developmental cycle of Plasmodium falciparum. PLoS Biol. 1: 85-99.

Carlton, J.M., Angiuoli, S.V., Suh, B.B., Kooij, T.W., Pertea, M., Silva, J.C., Ermolaeva, M.D., Allen, J.E., Selengut, J.D., Koo, H.L., et al. 2002. Genome sequence and comparative analysis of the model rodent malaria parasite Plasmodium yoelii yoelii. Nature 419: 512519.

Eddy, S.R. 2002. A memory-efficient dynamic programming algorithm for optimal alignment of a sequence to an RNA secondary structure. BMC Bioinformatics 3: 18.

Escalante, A.A. and Ayala, F.J. 1994. Phylogeny of the malarial genus Plasmodium, derived from rRNA gene sequences. Proc. Natl. Acad. Sci. 91: 11373-11377.

Fu, L.H., Wang, X.F., Eyal,Y., She, Y.M., Donald, L.J., Standing, K.G., and Ben-Hayyin, G. 2002. A selenoprotein in the Plant kingdom: Mass spectrometry confirms that an opal codon (UGA) encodes selenocysteine in Chlamydomonas reinhardtii glutathione peroxidase. J. Biol. Chem. 277: 25983-25991.

Gardner, M.J., Hall, N., Fung, E., White, O., Berriman, M., Hyman, 
R.W., Carlton, J.M., Pain, A., Nelson, K.E., Bowman, S., et al. 2002. Genome sequence of the human malaria parasite Plasmodium falciparum. Nature 419: 498-511.

Gautheret, D. and Lambert, A. 2001. Direct RNA definition and identification from multiple sequence alignments using secondary structure profiles. J. Mol. Biol. 313: 1003-1011.

Gladyshev, V.N., Krause, M., Xu, X.-M., Korotkov, K.V., Kryukov, G.V., Sun, Q.-A., Lee, B.J., Wootton, J.C., and Hatfield, D.L. 1999. Selenocysteine-containing thioredoxin reductase in C. elegans. Biochem. Biophys. Res. Commun. 259: 244-249.

Griffiths-Jones, S., Bateman, A., Marshall, M., Khanna, A., and Eddy, S.R. 2003. Rfam: An RNA family database. Nucleic Acids Res. 31: 439-441.

Hatfield, D.L. and Gladyshev, V.N. 2002. How selenium has altered our understanding of the genetic code. Mol. Cell. Biol. 22: 35653576.

Kohrle, J., Brigelius-Flohe, R., Bock, A., Gartner, R., Meyer, O., Flohe, L. 2000. Selenium in biology: Facts and medical perspectives. Biol. Chem. 381: 849-864.

Korotkov, K.V., Novoselov, S.V., Hatfield, D.L., and Gladyshev, V.N. 2002. Mammalian selenoprotein in which selenocysteine (Sec) incorporation is supported by a new form of Sec insertion sequence element. Mol. Cell. Biol. 22: 1402-1411.
Krol, A. 2002. Evolutionary different RNAmotifs and RNA-protein complexes to achieve selenoprotein synthesis. Biochimie 84: 765774.

Kryukov, G.V., Castellano, S., Novolselov, S.V., Lobanov, A.V., Zehtab, O., Guigo, R., and Gladyshev, V.N. 2003. Characterization of mammalian selenoproteomes. Science 300: 1439-1443.

Lambert, A., Lescure, A., and Gautheret, D. 2002. A survey of metazoan selenocysteine insertion sequences. Biochimie 84: 953-959.

Lowe, T.M. and Eddy, S.R. 1997. tRNAscan-SE: A program for improved detection of transfer RNA genes in genomic sequence. Nucleic Acids Res. 25: 955-964.

Novoselov, S.V., Rao, M., Onoshko, N.V., Zhi, H., Kryukov, G.V., Xiang, Y., Weeks, D.P., Hatfield, D.L., and Gladyshev, V.N. 2002. Selenoproteins and selenocysteine insertion system in the model plant cell system, Chlamydomonas reinhardtii. EMBO J. 21: 36813693.

Osaka, T., Beika, A., Hattori, A., Kohno, Y., Kato, K.H., and Mizutani, T. 2003. The protozoa dinoflagellate Oxyrrhis marina contains selenoproteins and the relevant translation apparatus. Biochem. Biophys. Res. Commun. 300: 236-240.

Rich, S.M. and Ayala, F.J. 2000. Population structure and recent evolution of Plasmodium falciparum. Proc. Natl. Acad. Sci. 97: 69947001 . 

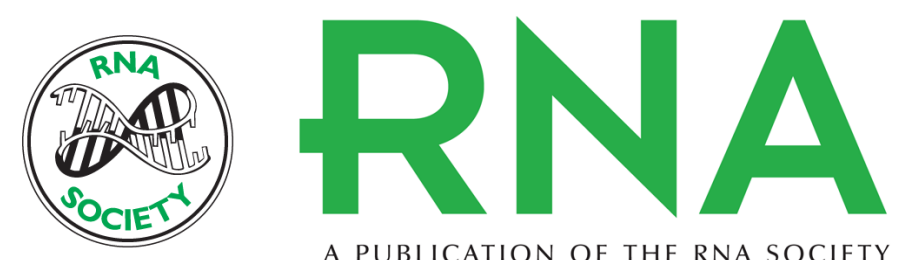

A PUBLICATION OF THE RNA SOCIETY

\section{A selenocysteine tRNA and SECIS element in Plasmodium falciparum}

TOBIAS MOURIER, ARNAB PAIN, BART BARRELL, et al.

RNA 2005 11: 119-122

References This article cites 22 articles, 8 of which can be accessed free at:

http://rnajournal.cshlp.org/content/11/2/119.full.html\#ref-list-1

License

Email Alerting Receive free email alerts when new articles cite this article - sign up in the box at the Service top right corner of the article or click here. 